\title{
Editorial
}

\section{Wissenschaftlichkeit und Wirtschaftlichkeit in Orthopädie und Physiotherapie}

Jahrzehntelang hat die wissenschaftliche Medizin als einzige Disziplin gegolten, die von wirtschaftlichen und politischen Einflüssen unabhängig war. Medizin war reine Naturwissenschaft und darum wahr. Medizinische Wissenschaft präsentierte sich unabhängig von Weltanschauungen, Rassen, Religion und Politik, sozialen Strukturen und ökonomischen Zwängen. Denken und Handeln des Arztes schien über allem zu stehen, denn er orientierte sich an den wissenschaftlichen Erkenntnissen über die Naturgesetze des menschlichen Körpers und diese schienen unstreitig: so wie es die Wissenschaft lehrte, so wurde diagnostiziert und therapiert.

Dieses Glashaus hat in den 60er Jahren einen ersten Sprung bekommen, als postuliert wurde, daß die Entstehung von Krankheiten von politischen Systemen abhängig und daran die Diagnostik auszurichten sei. Jetzt zeigt sich ein weiterer Riß in der Fassade: die Behandlung von Krankheiten ist nicht mehr nur wissenschaftlichen Erkenntnissen, sondern auch - und gelegentlich vor allem wirtschaftlichen Zwängen unterworfen.

„Die Kostenexplosion“ im Gesundheitswesen fordert ihren Tribut. Gemeint sind die Bemühungen „wirksame von unwirksamen "Behandlungsmethoden zu unterscheiden. Ein Vorhaben, dem sich manche Kostenträger, Ärzte und Verbände unter dem Aspekt wirtschaftlicher Zwänge sehr oberflächlich und damit unwissenschaftlich angenommen haben.

Was ist „medizinisch notwendig“? - wird zunehmend gefragt und eine Unterscheidung in medizinisch notwendige, ärztliche empfehlenswerte, ärztlich vertretbare und medizinisch machbare Leistungen empfohlen (Krimmel 1997). Die Diskussion über den Handlungsspielraum des Arztes wird offenbar ausschließlich unter den Aspekten der derzeit herrschenden ökonomischen Bedingungen geführt. Dabei geraten wissenschaftliche Erkenntnisse der Medizin und damit auch das System der naturwissenschaftlich begründeten Diagnostik und Therapie von Erkrankungen schnell unter die Räder.

Auch die Orthopädie wurde davon nicht verschont. Unter dem Budgetzwang bei der ambulanten Versorgung haben sich Kreise gebildet, die die Physiotherapie bei zahlreichen orthopädischen und auch neurologischen Erkrankungen schlichtweg als unwirksam und nicht indiziert bezeichnet haben (,Leitlinien zur rationalen Verordnung von Heilmitteln“, KV Südwürttemberg 1996).

Z. Orthop. 134 (1997) 1-2

(C) 1997 F. Enke Verlag Stuttgart
Wenngleich der wirtschaftliche Druck verständlich ist, Wirtschaftlichkeit sollte und darf nicht mit Wissenschaftlichkeit verwechselt werden. Wenn sich medizinische Behandlungsmaßnahmen im Rahmen des Budgetzwanges als unwirtschaftlich herausstellen, so sind sie deswegen noch lange nicht unwirksam. Es ist zwar richtig, daß therapeutische Prinzipien immer wieder einer kritischen Überprüfung bedürfen. Aber genauso richtig ist, daß der erforderliche ökonomische Ansatz für die Medizin von morgen in jedem Fall einer wissenschaftlichen Überprüfung bedarf.

Dies gilt natürlich auch für die konservative Orthopädie und die Physiotherapie. So erschien z. B. in der Zeitschrift Lancet ein Artikel, der die Wirksamkeit der Manualtherapie und der Diathermie vergleicht und keine Unterschiede findet. Die Autoren kommen daraufhin zu dem Schluß, $\mathrm{da} ß$ beide Maßnahmen durch ihren Placeboeffekt wirken (Gibson et al., 1985). Dies scheint manche Argumentationen zu unterstützen, daß Physiotherapie zwar medizinisch machbar, aber nicht notwendig sei. Es gibt aber auch Mitteilungen, bei denen die Manualtherapie und die Anwendung physikalischer Methoden mit der Effizienz einer rein ärztlichen Behandlung verglichen wurden. Dabei schnitten diese Methoden besser ab als die ausschließlich ärztliche Betreuung (Koes et al., 1992).

Widersprüchliche Ergebnisse über die Effizienz physiotherapeutischer und physikalischer Methoden gibt es in großem Umfang. Am Kniegelenk wurde eine Beschleunigung der postoperativen Verläufe durch Physiotherapie nachgewiesen (Moffet 1994). Demgegenüber stehen Untersuchungen, die eine solche positive Wirkung nicht nachweisen konnten (Birch et al., 1993). Ähnliche Diskrepanzen gibt es für die Wirksamkeit der Physiotherapie bei der idiopathischen Skoliose (Keim 1982 bzw. Niethard und Schneider 1991) oder bei der infanzilen Zerebralparese (Bower und McLellan 1992 bzw. Vojta 1984).

Diese Liste ist bei weitem nicht vollständig. Aber die Liste von Widersprüchlichkeiten in der Medizin läßt sich auf alle chirurgischen Interventionen, auf die Effizienz medikamentöser Behandlungen und das ärztliche Handeln schlechthin ausdehnen (Skrabanek und McCormick 1991).

Die z. T. widersprüchlichen Ergebnisse der Physiotherapie sind vor allem auf methodologische Mängel zurückzuführen. Dies hat eine Analyse von physiotherapeutischen Studien in den Niederlanden gezeigt (Bouter et al., 1992). Dort werden die Ergebnisse physiotherapeutischer Maßnahmen in großem Umfang überprüft. Dort sind aber die Physiotherapeuten auch akademisiert. 
Damit sind in der Bundesrepublik Deutschland die Ärzte selbst und ganz direkt angesprochen. Die Überprüfung und Qualitätssicherung ärztlicher Maßnahmen liegt in deren eigenen Händen. Bezüglich der Physiotherapie sind die Orthopäden daher aufgerufen, Beleg zu führen und sich stärker mit der Effizienz konservativer Maßnahmen zu beschäftigen. Die Zeitschrift für Orthopädie will diese Diskussion gern aufgreifen und hofft auf Beiträge aus diesem Bereich. Denn es ist kaum vorstellbar, daß Orthopädie und Physiotherapie den Boden der über 100 Jahre alten Zusammenarbeit, der wissenschaftlichen Erkenntnisse und des Erfahrungsaustausches verlassen. Nur durch wissenschaftlich begründete Zusammenarbeit profitiert der Patient und das sollte doch im Mittelpunkt aller ärztlicher Überlegungen stehen.

\section{Literatur}

Birch, N. C., C. Sly, S. Brooks, D. P. Powles: Anti-inflammatory Drug Therapy after Arthroscopy of the Knee. J. Bone Jt. Surg. 75-B (1993) 650-652

Bouter, L. M., H. Beckerman, G. M.J. G. von der Heijden, B. W. Koes, R. A. de Bie: Effectiviteit von fysiotherapie; een samenvatting van 9 meta-analyses. Ned. Tijdschr. Geneeskd 136 (1992) 1058-1061

Bower, E., D. L. McLellan: Evaluating Therapy in Cerebral Palsy. Child. care, health and development 20 (1994) 409-419
Gibson, T., R. Grahame, J. Harkness, P. Woo, P. Blagrave, R. Hills: Controlled Comparison of Short-wave Diathermy Treatment with Osteopathic Treatment in Non-specific Low Back Pain. Lancet (1985) $1258-1260$

Keim, H.: The Adolescent Spine. Springer, New York, Heidelberg, Berlin 1982

Krimmel, H.: „Was ist medizinische notwendig“? Dtsch. Ärzteblatt 94 (1997) B-97

Koes, B. W., L. M. Bouter, H. van Mameren, A. H. M. Essers, G. M. J. R. Verstegen, D. M. Hofhuizen, J. P. Houben, P. G. Knipschild: The Effectiveness of Manual Therapy, Physiotherapy, and Treatment by the General Practitioner for Nonspecific Back and Neck Complaints. Spine 17 (1992) 28-35

Moffet, H., C. L. Richards, F. Malouin, G. Bravo, G. Paradis: Early and Intensive Physiotherapy Accelerates Postarthroscopic Meniscecktomy: Results of a Randomized Controlled Study. Arch. Phys. Med. Rehabil. 75 (1994) 415-426

Niethard, F. U., E. Schneider: Results of a Neurophysiological Exercise Program in Children with Idiopathic Scoliosis. In: Scoliosis Update Basic Research and Clinical Concepts. Hrsg.: Canadell, J., F. Forriol, J. A. Cara: Edificio de biblioteca, Navarra 1991

Skrabanek, P., J. McCormick: Torheiten und Trugschlüsse in der Medizin. Verlag Kirchheim Mainz 1991

Vojta, $V$.: Die zerebralen Bewegungsstörungen im Säuglingsalter. Enke, Stuttgart 1984 